17. During this period ETAT+protocols were implemented through a three-month training programme. Data collected included demographics, symptoms at presentation, quality of care and outcome.

Results The results are as presented in the table 1 .

\begin{tabular}{llllll} 
Abstract G283(P) Table 1 & \multicolumn{7}{l}{} \\
\hline & March & April & May & June & Total \\
\hline $\begin{array}{l}\text { No. of admissions } \\
\text { Met 'complicated malaria' criteria }\end{array}$ & 45.2 & 60 & 78 & 124 & 315 \\
$\begin{array}{l}\text { (\%) } \\
\text { Median age at admission (months) }\end{array}$ & 31 & 24 & 15.5 & 24 & \\
No. requiring blood transfusion & 11 & 7 & 22 & 48 & \\
No. with severe respiratory & 9 & 6 & 20 & 19 & \\
distress & & & & & \\
No. with convulsions requiring & 4 & 10 & 17 & 18 & \\
treatment & & & & & \\
No. with hypoglycaemia & 8 & 8 & 12 & 18 & \\
Correct dose anti-malarial (\%) & 94.3 & 91.7 & 96.2 & 92.7 & \\
Correct dose anti-convulsant (\%) & 50 & 80 & 100 & 100 & \\
Mortality & 5 & 2 & 3 & 7 & \\
& $(9.4 \%)$ & $(3.3 \%)$ & $(3.8 \%)$ & $(5.6 \%)$ & \\
\hline
\end{tabular}

Conclusion This hospital was supported by an international non-government organisation (NGO) at the time of ETAT +implementation, and as such baseline mortality rate was lower than that of unsupported government hospitals. There were challenges of collaberative working. Overall mortality was reduced after the implementation of ETAT+, despite a significant rise in complicated malaria admissions correlating with the start of rainy season. There was a reduction in the number of uncomplicated malaria cases admitted and treated with IV therapy, thus reducing inappropriate use of limited medication. There was an improvement in anti-convulsant prescribing after implementation. Paediatric emergency care training is essential to ensure continued delivery of life-saving treatments, particularly in the context of withdrawal of NGO support.

\section{G284(P) A RETROSPECTIVE STUDY INVESTIGATING MALNUTRITION IN HIV POSITIVE CHILDREN IN SIERRA LEONE}

${ }^{1} \mathrm{~J}$ Finch, ${ }^{2} \mathrm{~L}$ Benton, ${ }^{2} \mathrm{P}$ Winyard, ${ }^{2} \mathrm{H}$ Bedford, ${ }^{2} \mathrm{~V}$ Palit. ${ }^{1}$ Institute of Child Health, University College London, London, UK; ${ }^{2}$ Institute of Child Health, University College London, Great Ormond Street, London, UK

\subsection{6/archdischild-2018-rcpch.276}

Aims To determine the prevalence of acute malnutrition, stunting and underweight in HIV infected children in an urban outpatient clinic in Sierra Leone, to establish risk factors predisposing to malnutrition, determine the outcome of these patients after 18 months follow-up, and make recommendations to minimise malnutrition in this population.

Methods A retrospective study of 409 HIV positive paediatric patients managed in an urban hospital in Sierra Leone. Data was gathered from notes at enrolment, and at 3, 6, 9, 12, 15 and 18 months after enrolment.

Results Between enrolment and 18 months the malnutrition prevalence improved significantly; acute malnutrition prevalence $54.0 \%$ to $7.1 \%$, stunting $53.9 \%$ to $8.0 \%$, and underweight $62.6 \%$ to $28.9 \%$. Logistic regression analysis showed a significant association between chronic diarrhoea (OR 4.1, CI: 2.48 to 6.8), cough (OR 3.0, CI: 2.19 to 4.09), tuberculosis (OR 1.4, CI: 1.04 to 1.8 ), oral thrush (OR 4.1, CI: 2.44 to 6.22), recurrent infection (OR 2.7, CI: 1.43 to 5.09), advanced stage (OR 7.4, CI: 1.05 to 80.1) and malnutrition. Protective factors against malnutrition included higher CD4 counts (CD4 >500 OR 0.27, CI: 0.13 to 0.60), female sex (OR 0.45, CI: 0.35 to 0.58), and ARVs (OR 0.18, CI: 0.11 to 0.30$)$. Multivitamin supplementation was not protective. At 18 months 23.0\% were lost to follow-up, 5.7\% died. A higher prevalence of malnutrition was observed in the lost to follow-up group (acute malnutrition $78.3 \%$ vs $54.0 \%$, underweight $70.4 \%$ vs $60.6 \%$, stunting $65.8 \%$ vs $53.9 \%$ ) and the died group (stunting $66.6 \%$ vs $53.9 \%$, underweight $77.8 \%$ vs $60.6 \%)$.

Conclusion Most patients were acutely and chronically malnourished at presentation. A significant association was observed between opportunistic infection, advanced stage and malnutrition. Malnutrition at presentation was associated with increased mortality. Initiation of ARVs, antimicrobials and RUTF improved malnutrition prevalence. Focusing on antenatal screening to reduce transmission and earlier intervention with ARVs, antimicrobials and nutritional supplementation would improve outcomes and mortality rates. Since this study, the Ebola epidemic has diverted already limited resources away from existing public health programmes, outcomes are now likely to be even worse than at the time of data collection.

\section{G285(P) CASES WITHOUT BORDERS: CAN ONLINE DISCUSSION OF CLINICAL CASES BETWEEN HIGH AND LOW RESOURCE COUNTRIES ENHANCE LEARNING IN PAEDIATRICS?}

${ }^{1,2,3} \mathrm{C}$ Crehan, ${ }^{4,5} \mathrm{M}$ Lee. ${ }^{1}$ Great Ormond Street Institute of Child Health, University College London, London, UK; ${ }^{2}$ Paediatric Department, North Middlesex Hospital NHS Trust, London, UK; ${ }^{3}$ Paediatric Department, Zomba Central Hospital, Zomba, Malawi; ${ }^{4}$ Paediatric Department, Northwick Park Hospital, London North West Healthcare NHS Trust, London, UK; ${ }^{5}$ Liberia Mission, Médecins Sans Frontières, Operational Centre Paris, Liberia

\subsection{6/archdischild-2018-rcpch.277}

Background and aims Gaining exposure to global child health can be difficult to organise both financially and logistically. 'Cases without borders' aims to evaluate the perceived learning benefits of an online case-exchange programme comparing clinical management between contrasting settings.

Methods One hour online sessions were carried out over five months between two paediatric departments; a Malawian district hospital and a UK teaching hospital.

Clinicians, Nurses and Students from both settings were invited to attend sessions facilitated by a UK registrar in each facility, using Skype or Whatsapp. Proformas divided down the middle, were circulated to participants in advance. The clerking and management of a case from each setting was entered on one side and emailed to the whole group. During the online presentation and discussion, the other hospital considered how that case would be managed in their alternative setting, completing the other side of the proforma. Feedback was collected using an unvalidated perceived learning questionnaire devised by the facilitators using 10 questions on a 5point likert scale. 
Results 6 exchange sessions were held covering 12 paired paediatric cases (table 1).

Feedback was collected from 13 participants (7 Malawi, 6 UK). Mean score of all 10 questions was 4.26 out of 5 for all responders (4.3 for Malawi, 4.2 for UK), indicating perceived learning was high and similar for both groups. There was no significant difference in mean scores between the groups ( $>0.05$ using Mann-Whitney-U) for all feedback questions.

Conclusions Cases without borders was perceived to enhance learning across settings, equally benefiting both sides, suggesting it is a suitable model for telemedicine programmes. Qualitative feedback suggests potential for increased interest and participation in evidence-based medicine and global child health in both contexts. Future iterations would benefit from an improved internet connexion and a larger feedback sample using a validated feedback tool.

\begin{tabular}{ll} 
Abstract G285(P) Table 1 Malawi & \\
\hline Organophosphate poisoning & Asthma exacerbation \\
\hline Malaria, Malnutrition and dehydration & Diabetic keto-acidosis \\
PJP Pneumonia & Sickle-Cell Painful crisis \\
Congenital syphilis & Cerebral Malaria \\
Prematurity with RDS & Sepsis and metabolic condition \\
Rheumatic fever and Sydenham's chorea & Bronchiolitis \\
\hline
\end{tabular}

\section{G286(P) THE ACCEPTABILITY, FEASIBILITY AND USABILITY OF THE NEOTREE APPLICATION IN MALAWI: AN INTEGRATED DATA COLLECTION, CLINICAL MANAGEMENT AND EDUCATION MHEALTH SOLUTION TO IMPROVE QUALITY OF NEWBORN CARE AND THUS NEWBORN SURVIVAL IN HEALTH FACILITIES IN RESOURCE-POOR SETTINGS}

${ }^{1} \mathrm{C}$ Crehan, ${ }^{1,2} \mathrm{E}$ Kesler, ${ }^{3} \mathrm{~B}$ Nambiar, ${ }^{4} \mathrm{O}$ Dube, ${ }^{5,6} \mathrm{~N}$ Lufesi, ${ }^{7} \mathrm{M}$ Giaconne, ${ }^{8} \mathrm{C}$ Normand, ${ }^{1} \mathrm{M}$ Heys. ${ }^{1}$ Great Ormond Street Hospital Institute of Child Health, University College London, UK; ${ }^{2}$ University of Pennsylvania, USA; ${ }^{3}$ Institute for Global Child Health, University College London, UK; ${ }^{4}$ Paediatric Department, College of Medicine, University of Malawi, Blantyre, Malawi; ${ }^{5}$ Ministry of Health Acute Respiratory Illness Unit, Government of Malawi, Lilongwe, Malawi; ${ }^{6}$ Paediatrics and Child Health Association (PACHA), Blantyre, Malawi; ${ }^{7} U$ biqueworks Ltd, London, UK; ${ }^{8}$ Spinspire Consulting Ltd, London, UK

\subsection{6/archdischild-2018-rcpch.278}

Background An estimated 70\% of newborn lives could be saved globally if evidence-based interventions were successfully implemented. The 'NeoTree' application (app) addresses the need for quality care for sick newborns in resource-poor settings, particularly where care is nurse-led, by providing an integrated electronic platform for three functions;

1) immediate digital data capture on admission,

2) clinical decision support according to evidence-based clinical guidelines, and

3) newborn education. Here, we develop the first two functions and test the acceptability, feasibility and usability of The NeoTree in a district hospital, Malawi.

Methods A mixed methods intervention development study was conducted with facility-based health care workers (HCWs) to co-develop and test the app. Methods included Focus Groups (FGs) exploring the acceptability and feasibility of digital health solutions, one-to-one usability workshops and a one-month ward-based usability study. Quantitative usability data (systems usability score - SUS) were collected before and after the ward study. Finally, end-line qualitative FGs were completed.

Results Digital aids, specifically the NeoTree were reported to be acceptable, feasible and a potential facilitator to quality newborn care. Identified factors predicted to aid the success of the NeoTree included a positive staff attitude, training, strong leadership, teamwork and staff engagement. SUS score was high at baseline (under workshop conditions) and following the ward study ( $>68 / 100$ average) and HCWs reported high perceptions of improved quality of care after embedding NeoTree into routine practice (see table 1).

Conclusions The NeoTree has the potential to address multiple Malawian and global policy directives around improving quality of care and outcomes for newborns. It is an acceptable, feasible and highly usable tool. Co-production and userfocused, mixed methods iterative development have been key to its success thus far. The next phase of development will focus on the educational function and data linkage with national systems.

$\begin{aligned} & \text { Abstract G286(P) Table } 1 \\ & \text { Neotree usability and quality improvement scores } \\ & (\mathrm{n}=13)\end{aligned}$
$\begin{aligned} & \text { SUS (calculated from } 10 \text { questions) } \\ & \text { PIQC (mean of } 11 \text { questions) }\end{aligned}$
$\begin{aligned} & \text { SUS=System usability score, PIQC=Perceived improved quality of care score } \\ & \text { G287(P) } \\ & \text { INVESTIGATING THE NEED, FEASIBILITY AND } \\ & \text { INTRODUCTION OF KETAMINE SEDATION IN A } \\ & \text { PAEDIATRIC EMERGENCY DEPARTMENT IN A } \\ & \text { RESOURCE-POOR SETTING }\end{aligned}$

1J Rasquinha, ${ }^{2} \mathrm{C}$ Ngulube, ${ }^{1} \mathrm{~J}$ Langton. ${ }^{1}$ Department of Paediatrics, Queen Elizabeth Central Hospital, Blantyre, Malawi; ${ }^{2}$ Department of Orthopaedics, Queen Elizabeth Central Hospital, Blantyre, Malawi

\subsection{6/archdischild-2018-rcpch.279}

Aims To evaluate the need for and practicalities of introducing ketamine sedation for manipulation of fractures, within a Paediatric Emergency Department in a resource-poor setting.

Methods Patient journeys were observed and 6 months of theatre records reviewed to evaluate the current fracture management pathway and determine a need for a change to service provision. In consultation with the orthopaedic team, a draft ketamine sedation policy was created, for fracture manipulation within the paediatric emergency department. Using one patient, this was then piloted to determine the practicalities of use.

Results Review of the current patient journey confirmed that for those requiring manipulation, this is lengthy process. Patients are discharged home and subsequently return for admission to the orthopaedic ward the following Monday or Friday. They are kept nil by mouth prior to general anaesthesia and following their procedure they return to the orthopaedic ward for recovery and review of post-procedure imaging. Over a 6 month period, 307 paediatric patients underwent a manipulation under anaesthesia in theatre, with a maximum of 16 performed on one list. Approximately half of these had fractures which would have been suitable for reduction under 